EPJ Web of Conferences 60, 20010 (2013)

DOI: $10.1051 /$ epjconf $/ 20136020010$

(C) Owned by the authors, published by EDP Sciences, 2013

\title{
Jet Energy Calibration in the CMS experiment
}

\author{
Denis Rathjens ${ }^{1, a}$, Henning Kirschenmann ${ }^{1}$, Christian Sander ${ }^{1}$, and Hartmut Stadie ${ }^{1}$ for the CMS Collaboration \\ ${ }^{1}$ Institute for Experimental Physics, University of Hamburg
}

\begin{abstract}
Jet reconstruction and calibration in the CMS experiment are complicated by the nonlinear response of the calorimeters and high pileup conditions. These difficulties are mitigated at CMS by utilising the particle flow approach. The jet energy calibration from data is summarized. It is performed with data samples collected in proton-proton collisions at a centre-of-mass energy of $8 \mathrm{TeV}$ corresponding to an integrated luminosity of $11 \mathrm{fb}^{-1}$. The final jet energy calibration is derived with dijet, $\gamma+$ jet and $\mathrm{Z}+$ jet events. Here, we focus on the estimation of the inter- $\eta$-calibration using dijet events and discuss the uncertainties on the jet energy corrections.
\end{abstract}

\section{Introduction}

Jets are a main signature in standard model and beyond standard model physics. In CMS, jets are clustered from particle flow (PF) [1] objects with the anti-kt [2] sequential recombination algorithm at a clustering radius of 0.5 . After reconstruction, jets need to be calibrated in order to measure at the same generator jet and reconstructed jet energy scale.

There is a difference between generator jet energy and reconstructed jet energy in simulation resulting from detector simulation effects like nonlinear calorimeter response, inactive material interactions and physical effects like neutrinos produced in the jet. There are residual differences in jet energy between simulation and data. These residual differences may be caused by time dependent detector response, due to e.g. radiation damage or jet energy resolution effects.

The corrections are dependent on a pileup in the event (CMS uses $\rho$, a measure for the pileup energy density in the event and substracts pileup on an event by event basis [3]), the angle towards the beampipe (CMS uses the pseudorapidity $\eta$ ) and a measure for the energy of the jet (CMS uses the transverse momentum $p_{T}$ ). The corrections are expected to be cylindrically symmetrical after accounting for dead calorimeter cells and other dysfunctional or inactive detector parts in the detector simulation.

The CMS collaboration uses a factorised correction scheme to calibrate the jet energy scale, correcting jets for pileup and then for $|\eta|$ - and $p_{T}$-dependence of the jet energy scale on simulated events. Finally, momentum conserving physics processes like dijet-, $\gamma+$ jet- and $\mathrm{Z}^{0}+$ jet-events are used to measure the jet energy scale on data. The absolute jet energy scale in the central part of the detector $(|\eta|<1.3)$ is determined with $Z^{0}+$ jet-events that have good energy resolution for the $Z^{0}$ in the $Z^{0} \rightarrow \mu^{+} \mu^{-}$ channel.

\footnotetext{
a e-mail: Denis.Rathjens@uni-hamburg.de
}

Here, the focus is on the inter- $\eta$-calibration relative to the central part of the detector to extend the residual calibration in $\eta$ and $p_{T}$. Dijet events are chosen for this analysis as they allow to access to a large phase space, especially at high momenta. The event selection, derivation of the jet energy scale, resulting correction factors as well as the impact on the overall uncertainties of the jet energy scale are shown.

\section{Dijet event selection and determination of the jet energy scale}

On simulated and data events, a loose jet identification entailing quality criteria on the jets is required for both leading jets in $p_{T}$. One of these jets needs to be in the central detector region $|\eta|<1.3$ and the jets have to point in opposite directions with a difference in $\phi$ of $\Delta \phi>2.7$ to enrich QCD dijet events. The events are binned in inclusive bins of the momentum of the third leading jet in $p_{T}$ relative to the mean momentum in the dijet event $\bar{p}_{T}=$ $\left(p_{T}^{j e t 1}+p_{T}^{j e t 2}\right) / 2$ so that $\alpha=p_{T}^{j e t 3} / \bar{p}_{T} \leq\{0.1,0.2,0.3,0.4\}$. This is done as a measure of final state radiation (FSR) unbalancing the event.

For MC the simulated QCD events are hadronized with PYTHIA [4]. On data, triggers on the $\bar{p}_{T}$ are required to fulfill standard quality criteria [5]. In total, $\approx 11 \mathrm{fb}^{-1}$ of data are used for the corrections shown here.

To determine the jet energy scale, an estimator of the energy response for data and simulation is used. The momentum projection fraction $\mathcal{R}_{M P F}[6]$ is defined as:

$$
\mathcal{R}_{M P F}=1+\frac{E_{T}^{\text {miss }} \cdot \cos \left(\Delta \phi\left(E_{T}^{\text {miss }}, j e t^{\text {tag }}\right)\right)}{p_{T, L 1 L 2 L 3}^{\text {tag }}}
$$

This is the projection of the missing energy $\left(E_{T}^{\text {miss }}\right)$ [7] on the tagged jet in order to estimate the mismeasurement in direction of the tagged jet. The missing energy is calculated as the vector sum of all reconstructed objects in the 
detector.

If both leading jets in transverse momentum point to the central detector region, both combinations of tag and probe enter into the calculation of the jet energy scale. Therefore, the combined correction factor of the central detector reference region needs to be at unity by design.

\section{Results}

The residual correction factors of the inter- $\eta$-calibration is shown in figure 1 . They have been determined with high statistical precision and low systematic uncertainties in the central part of the detector.

Low residual correction factors attribute to an accurate detector and physics simulation.

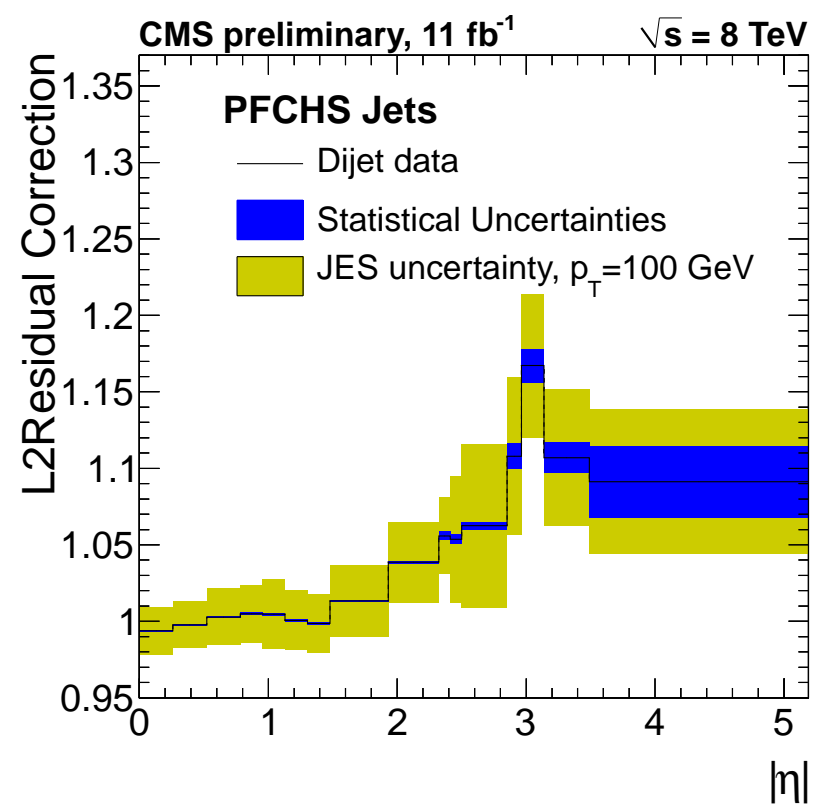

Figure 1. Inter- $\eta$-calibration constants for particle flow anti-kt 0.5 jets with charged hadron substraction with statistic and systematic uncertainties. [8] Less jets are recorded in the forward regions of the detector due to trigger requirements on the minimal transverse momentum of the forward jet. The main systematic uncertainty sources are the jet energy resolution and dependencies of the jet energy scale on the transverse momentum of the jets.

\section{Conclusion}

The total uncertainty on the jet energy scale is comprised of the uncertainties on the absolute scale of data derived on $Z^{0}+$ jet events, the relative scale of data derived on dijet events, the stability of these methods in terms of final state radiation (called extrapolation uncertainty), the event by event pileup correction uncertainties, differences in description of jet flavour and hadronization between PYTHIA and Herwig++ generators (called Jet flavor uncertainty) and the stability of the measured jet energy scale as a function of time.

In the figures 2 and 3 the total uncertainties and their components are shown.

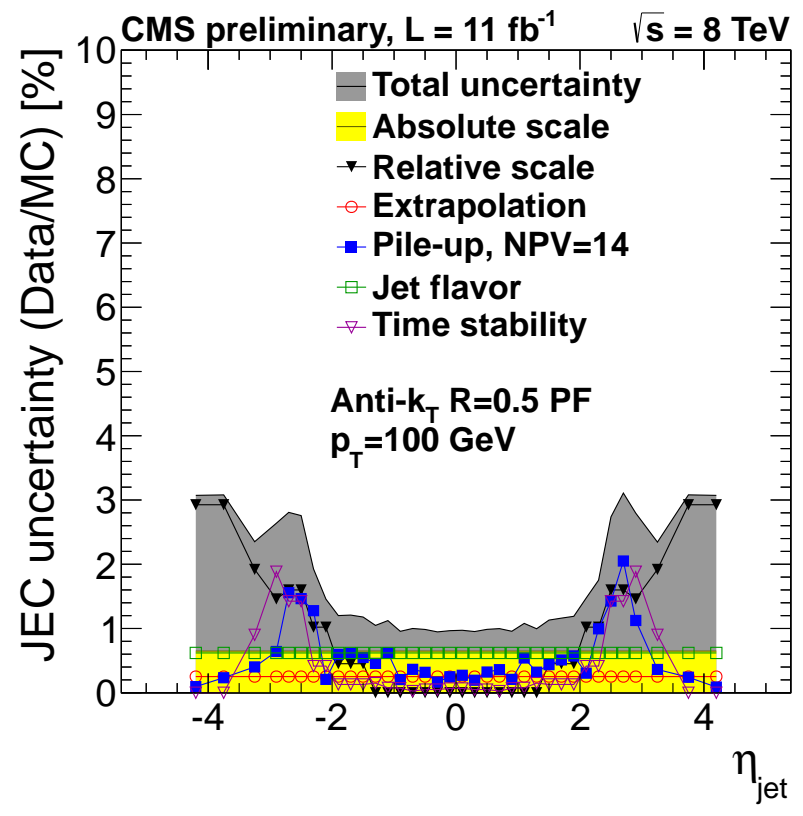

Figure 2. Uncertainty sources for the jet energy scale of ak5 particle flow jets at a transverse momentum of $100 \mathrm{GeV}$. [8]

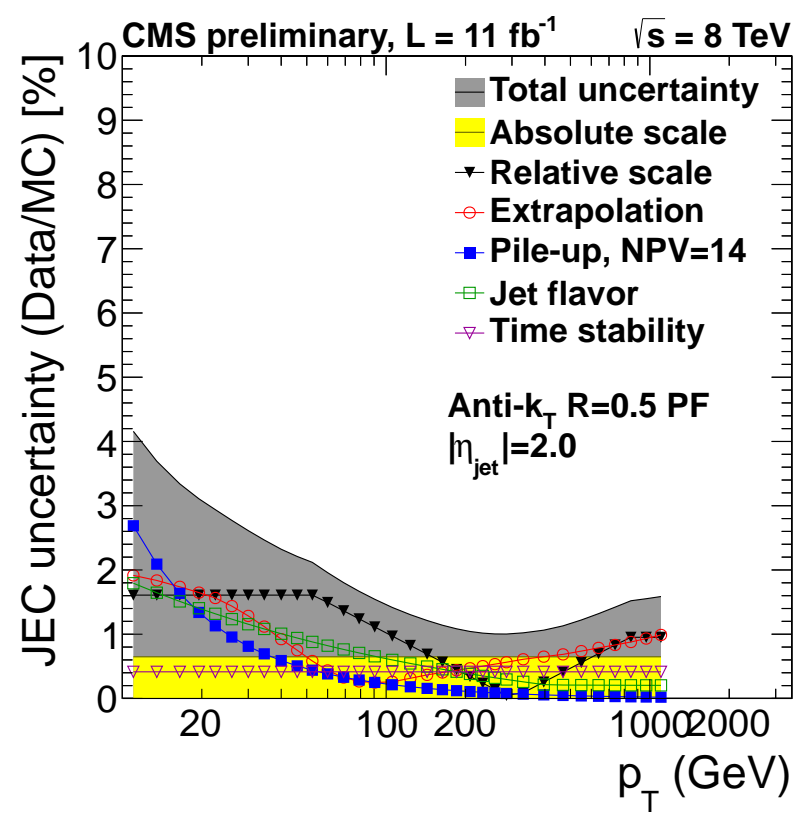

Figure 3. Uncertainty sources of the jet energy scale of ak5 particle flow jets in the endcap detector region. [8]

The relative scale as the inter- $\eta$-calibration contributes minimally to the uncertainties in the central part of the detector with less than $0.1 \%$ uncertainty and is neglected in favor of the absolute scale uncertainty up to $|\eta| \leq 1.3$ that has to be applied on the full range in $\eta$. The correction factors in the endcap of the detector increase to up to $10 \%$ with uncertainties of up to $1.7 \%$. In the forward detector regions, larger correction factors of up to $16 \%$ (see figure 3 ) have to be applied. At low energies and in very forward detector regions, higher uncertainties of up to $3 \%$ are 
caused by high trigger tresholds and therefore low statistics as well as large uncertainties on the measurements of the detector resolution.

\section{References}

[1] [CMS Collaboration], CMS-PAS-PFT-10-002.

[2] M. Cacciari, G. P. Salam and G. Soyez, JHEP 0804 (2008) 063 [arXiv:0802.1189 [hep-ph]].
[3] M. Cacciari and G. P. Salam, Phys. Lett. B 659 (2008) 119 [arXiv:0707.1378 [hep-ph]].

[4] T. Sjostrand, S. Mrenna and P. Z. Skands, JHEP 0605 (2006) 026 [hep-ph/0603175].

[5] [CMS Collaboration], CMS-PAS-JME-10-003.

[6] F. Abe et al. [CDF Collaboration], Phys. Rev. Lett. 69 (1992) 2896.

[7] [CMS Collaboration], CMS-PAS-JME-12-002.

[8] [CMS Collaboration], CERN-CMS-DP-2013-011 